\title{
Modulation of flavocytochrome $b_{2}$ intraprotein electron transfer via an interdomain hinge region
}

\author{
R. Eryl SHARP* ${ }^{\star}$, Stephen K. CHAPMAN $\dagger$ and Graeme A. REID*\$ \\ Edinburgh Centre for Molecular Recognition, University of Edinburgh, *Institute of Cell and Molecular Biology and †Department of Chemistry, Kings Buildings, \\ Mayfield Road, Edinburgh EH9 3JR, Scotland, U.K.
}

The two domains of flavocytochrome $b_{2}$ are connected by a typical hinge peptide. To probe the role of the hinge in modulating the efficiency of intraprotein electron transfer between these two domains, a number of mutant enzymes with truncated hinge regions were previously constructed and characterized [Sharp, Chapman and Reid (1996) Biochemistry 35, 891-899]. In the present study two mutant enzymes with elongated hinge regions have been constructed ( $\mathrm{H} / 3$ and $\mathrm{H} / 6)$ to further our understanding of the controlling influence of hinge length and primary structure on intraprotein electron transfer. Modification of the hinge had little effect on the lactate dehydrogenase activity of the enzyme, as was evident from steady-state experiments using ferricyanide as electron acceptor and from pre-steady-state experiments monitoring flavin reduction. However, the hinge insertions lowered the enzyme's effectiveness as a cytochrome $c$ reductase. This effect results from a defect at the first interdomain electron-transfer step $\left(\mathrm{FMNH}_{2} \rightarrow\right.$ haem electron transfer), where the rate constants for haem reduction in $\mathrm{H} I 3$ and $\mathrm{H} I 6$ were 50and 100-fold lower than the corresponding value for the wildtype enzyme. Preservation of structural integrity within the hinge region is apparently essential for efficient intraprotein electron transfer.

\section{INTRODUCTION}

Saccharomyces cerevisiae flavocytochrome $b_{2}$ (L-lactate cytochrome $c$ oxidoreductase, EC 1.1.2.3) is a soluble component of the mitochondrial intermembrane space [1], where it catalyses dehydrogenation of L-lactate to pyruvate with subsequent electron transfer to cytochrome $c$ [2]. The enzyme is a homotetramer of subunit molecular mass $57500 \mathrm{Da}$ [3]. The crystal structure of flavocytochrome $b_{2}$ solved to $0.24 \mathrm{~nm}$ resolution [4] shows that each subunit is composed of two distinct domains: a $10 \mathrm{kDa} \mathrm{N}$ terminal domain containing protohaem IX and a $47 \mathrm{kDa} \mathrm{C}$ terminal domain containing FMN non-covalently bound at the active site for L-lactate dehydrogenation (Figure 1). The physiological pathway for electron transfer is: L-lactate $\rightarrow \mathrm{FMN} \rightarrow$ haem $\rightarrow$ cytochrome $c$, with no detectable electron transfer directly from the FMN in intact flavocytochrome $b_{2}$ to cytochrome $c[6,7]$.

The two domains of each flavocytochrome $b_{2}$ subunit are connected by a typical hinge peptide sequence that contains proline, glycine and various charged residues (Figure 2). The presence of the hinge region is physically required to covalently link the two domains and maintain them in close proximity with one another. Kinetic and spectroscopic experiments with the independently expressed and purified haem and FMN domains show that no detectable electron transfer occurs from reduced FMN to oxidized $b_{2}$ haem and the two domains do not appear to interact in solution [8]. It has also been proposed that the hinge region confers domain mobility, allowing movement of the cytochrome domain with respect to the FMN domain [9]. This proposal is supported by observations from X-ray crystallography [4] and NMR spectroscopy [10].

In order to investigate the effect of disrupting structural integrity within the hinge region on intraprotein electron transfer
(FMN $\rightarrow$ haem electron transfer), a number of hinge-deletion mutant enzymes have been previously constructed and characterized. These are: $\mathrm{H} \Delta 3$ [11], $\mathrm{H} \Delta 6$ and $\mathrm{H} \Delta 9$ [12], with 3,6

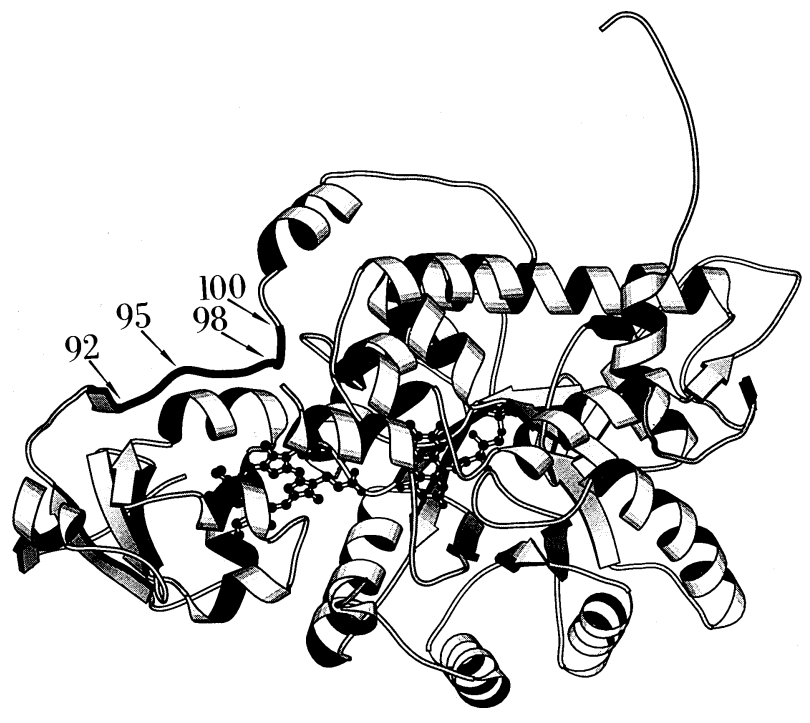

Figure 1 Graphical representation of a single wild-type flavocytochrome $b_{2}$ subunit

This diagram was generated using the MOLSCRIPT molecular graphics program [5]. $\alpha$-Helices and $\beta$-sheets are shown as ribbons, the remaining $\alpha$-carbon backbone is shown as a wire. Atoms depicted in ball and stick format represent the prosthetic groups. The numbered arrows indicate the end-points of insertions and deletions described in this paper.

Abbreviations used: KIE, kinetic isotope effect; cyt $c$, cytochrome $c ; k^{F}$, pre-steady-state rate constant for flavin reduction; $k^{H}$, pre-steady-state rate constant for haem reduction; $K_{m}{ }^{F}$, apparent $K_{m}$ for pre-steady-state flavin reduction; $K_{m}{ }^{H}$, apparent $K_{m}$ for pre-steady-state haem reduction.

+ Present address: Johnson Research Foundation, Department of Biochemistry and Biophysics, B501 Richards Building, University of Pennsylvania, Philadelphia, PA 19104, U.S.A.

$\S$ To whom correspondence should be addressed at, ICMB, Darwin Building, University of Edinburgh, Mayfield Road, Edinburgh EH9 3JR, Scotland, U.K 


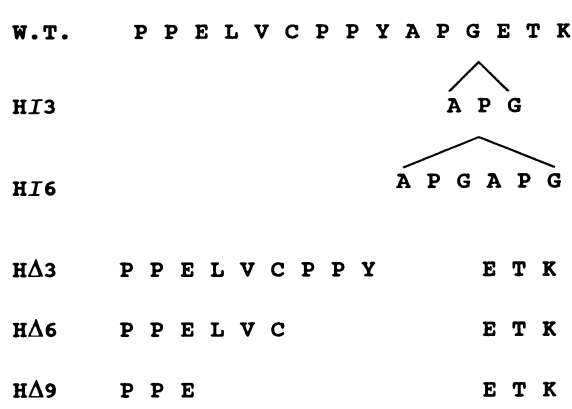

Figure 2 Construction of hinge-modified flavocytochromes $\boldsymbol{b}_{2}$

The amino acid sequence of the interdomain hinge region is shown, from Pro-89 to Lys-103 of the wild-type enzyme. The H/3 and H/6 enzymes have three and six amino acids inserted at the indicated position in the primary structure. The gaps in the sequence indicate the amino acid residues deleted from the hinge sequence in the $H \Delta 3, H \Delta 6$ and $H \Delta 9$ enzymes.

and 9 amino acid residues deleted from the hinge region respectively (Figures 1 and 2). Kinetic experiments showed that all these enzymes were defective in intraprotein electron transfer, with a direct correlation between the degree of truncation and the effectiveness of intraprotein electron transfer. $\mathrm{H} \Delta 3, \mathrm{H} \Delta 6$ and $\mathrm{H} \Delta 9$ had $\mathrm{FMNH}_{2} \rightarrow$ haem electron transfer rate constants which were 16-, 50- and 170-fold lower respectively than the value for the wild-type enzyme [10,11]. However, all these hinge-truncated enzymes remained good L-lactate dehydrogenases, compared with the wild-type enzyme. Clearly, the functionality of intraprotein electron transfer in flavocytochrome $b_{2}$ is severely compromised by truncation of the interdomain hinge region.

The aim of this study was to examine the effect of elongating the hinge region of flavocytochrome $b_{2}$ on the efficiency of intraprotein electron transfer. To this end we have constructed two further mutant enzymes, termed HI3 and HI6, with 3 and 6 amino acid residues inserted into the hinge region respectively (Figure 2). These insertions are positioned between residues 100 and 101 of the wild-type primary structure, the site defined structurally as the junction between the two domains [4]. We chose to insert repeats of the tripeptide motif Ala-Pro-Gly, as it occurs naturally in this portion of the hinge region (Figure 2) and these residues have small side chains and are commonly found in flexible regions of proteins. In addition, these insertions will have no effect upon net charge.

\section{MATERIALS AND METHODS}

\section{DNA manipulation, strains and growth}

Site-directed mutagenesis was performed by the Kunkel method of non-phenotypical selection [13] using the oligonucleotides $329 \mathrm{~N}$ (GTCCTCCTTATCGACCAGGAGCTCCTG GTGA) to construct $\mathrm{H} I 3$ and 330N (GTCCTCCTTATGCACCAGGAGCACCAGGAG CTCCTGGTGA) to construct HI6. Standard methods for growth of Escherichia coli, plasmid purification, DNA manipulation and transformation were performed as described by Sambrook et al. [14]. E. coli strain TG1 was used for expression of mutant flavocytochromes $b_{2}$.

\section{Enzymes}

Wild-type and hinge-insertion flavocytochromes $b_{2}$ overexpressed in $E$. coli were isolated from cells which had been stored at $-20{ }^{\circ} \mathrm{C}$. The purification procedure was as previously described [15], incorporating the modifications described by Sharp et al. [12]. Purified enzyme preparations were stored under a nitrogen atmosphere at $4{ }^{\circ} \mathrm{C}$ as precipitates in $70 \%\left(\mathrm{NH}_{4}\right)_{2} \mathrm{SO}_{4}$, under these conditions the enzymes retained full activity for several weeks. Alternatively, for long-term storage, concentrated aliquots $(>100 \mu \mathrm{M})$ of oxidized, $\left(\mathrm{NH}_{4}\right)_{2} \mathrm{SO}_{4}$-free enzymes were snap-frozen and stored in liquid nitrogen. Under these conditions, the enzymes retained full activity indefinitely. Enzyme concentrations were calculated using previously published absorption coefficients [16].

\section{Kinetic analysis}

All kinetic experiments were performed at $25 \pm 0.1^{\circ} \mathrm{C}$ in $10 \mathrm{mM}$ Tris $/ \mathrm{HCl}$ at $\mathrm{pH} 7.5, I=0.10 \mathrm{M}$ (ionic strength, $I$, adjusted to $0.10 \mathrm{M}$ by addition of $\mathrm{NaCl}$ ). Steady-state kinetic measurements involving the enzymic oxidation of L-lactate were performed using a Shimadzu UV2101PC spectrophotometer. Horse heart cytochrome $c$ (type VI, Sigma) or ferricyanide (potassium salt, $\mathrm{BDH}$ chemicals) was used as the electron acceptor, as previously described [17]. Pre-steady-state measurements involving single turnover of L-lactate were carried out with an Applied Photophysics SF.17 MV stopped-flow spectrofluorimeter, as previously described [17]. Flavin reduction was monitored at $438.3 \mathrm{~nm}$ (a haem isosbestic point) and haem reduction was monitored at either 423 or $557 \mathrm{~nm}$ (the measured rate constants were the same at both wavelengths). Collection and analysis of data were as previously described [17]. Values of kinetic constants were determined using non-linear regression analysis. Purification and preparation of $\mathrm{L}-\left[2-{ }^{2} \mathrm{H}\right]$ lactate [18] and measurements of kinetic isotope effect (KIE) values, using this substrate were as previously described [17].

\section{Measurement of redox potential}

The mid-point potentials of the haem groups of the hingeinsertion flavocytochromes $b_{2}$ were determined spectrophotometrically using a previously published redox potentiometry method [19]. The experiments were performed exactly as described before [12], except that the potentials were determined with a platinum electrode relative to a calomel cell. The potentials were corrected for the standard hydrogen electrode by addition of $242 \mathrm{mV}$ to the measured potential.

\section{RESULTS AND DISCUSSION}

\section{Effect of flavocytochrome $b_{2}$ hinge modifications on the catalysis of L-lactate dehydrogenation}

The catalytic cycle of flavocytochrome $b_{2}$ is shown diagrammatically in a linear form in Scheme 1. A step-wise description of the electron-transfer events occurring in this cycle are given in the legend to the Scheme. Starting with the fully oxidized enzyme, step 1 in Scheme 1 is reduction of the FMN prosthetic group by L-lactate. The kinetics of this reaction directly reflect the L-lactate dehydrogenase activity of the enzyme. Reduction of FMN by L$\left[2-{ }^{1} \mathrm{H}\right]$ lactate and $\mathrm{L}-\left[2-{ }^{2} \mathrm{H}\right]$ lactate for wild-type and hingemodified flavocytochromes $b_{2}$ was monitored directly, as described in the Materials and methods section. Pre-steady-state kinetic parameters for hinge-insertion enzymes are reported in Table 1 (top panel), where they are compared with previously reported values for hinge-deletion and wild-type flavocytochromes $b_{2}$.

The rate constants for FMN reduction $\left(k^{\mathrm{F}}\right)$ in the hingeinsertion enzymes, HI3 and HI6 are almost the same, within 

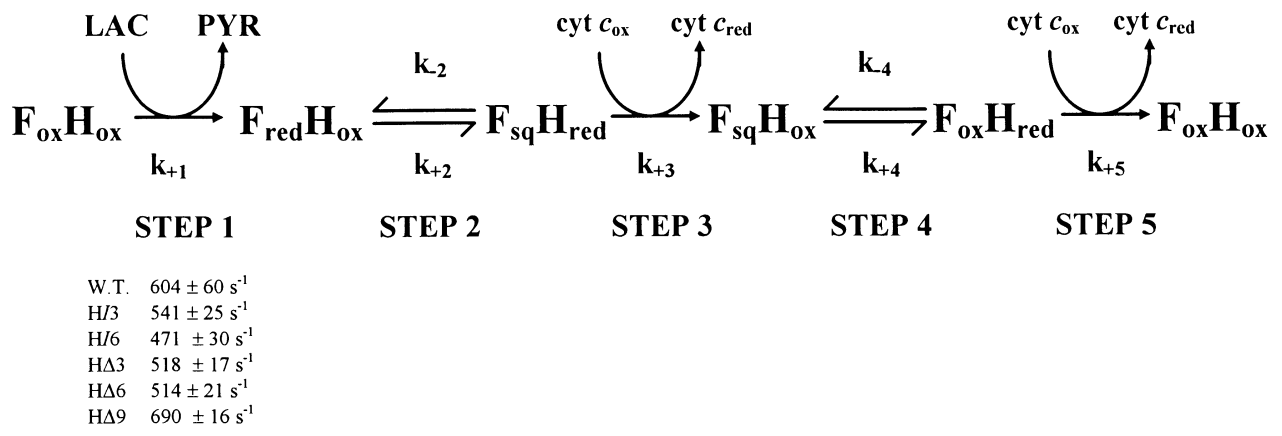

\section{STEP 2}

STEP 3

STEP 4

STEP 5

cyt $c$

$\begin{array}{lr}\text { W.T. } & 207 \pm 10 \mathrm{~s}^{-1} \\ \text { H/3 } & 28 \pm 1 \mathrm{~s}^{-1} \\ \text { H/6 } & 13 \pm 1 \mathrm{~s}^{-1} \\ \mathrm{H} \Delta 3 & 39 \pm 1 \mathrm{~s}^{-1} \\ \mathrm{H} \Delta 6 & 34 \pm 2 \mathrm{~s}^{-1} \\ \mathrm{H} \Delta 9 & 8 \pm 1 \mathrm{~s}^{-1}\end{array}$

\section{Scheme 1 Linear representation of the catalytic cycle of flavocytochrome $b_{2}$}

The redox states of cytochrome $c($ cyt $c)$ haem and flavocytochrome $b_{2}$ flavin $(F)$ and haem $(H)$ are indicated by the subscripts ' $0 x^{\prime}$ ' and 'red' for the oxidized and reduced forms respectively. The flavin semiquinone is shown as ' $F_{\text {sq }}$ '. The catalytic cycle is shown in a linearized form to facilitate presentation of the individual microscopic steps discussed. Steps on the catalytic cycle to which we wish to draw attention are as follows. (1) Oxidation of lactate to pyruvate and reduction of FMN; this contributes to rate limitation in the wild-type enzyme (especially the first haem reduction step; see the text for details). The rate constant for FMN reduction, within experimental error, for each enzyme is indicated. (2) Electron transfer from fully reduced FMN $\rightarrow$ haem, resulting in the semiquinone form of FMN and reduced haem (this is the rate-limiting step in all the hinge-modified enzymes, except $\mathrm{H} \Delta 3$ ). The rate constant for this step is indicated for the wild-type and hinge-modified enzymes. (4) Electron transfer from the flavin semiquinone to haem, resulting in fully oxidized FMN and reduced haem (this is the major rate-limiting step in the wild-type and $\mathrm{H} \Delta 3$ enzymes). The long arrow labelled 'cyt $c$ ' represents steady-state turnover of cytochrome $c$ by flavocytochrome $b_{2}$, the rate constants for the turnover by wild-type and hinge-modified enzymes are indicated.

Table 1 Pre-steady-state kinetic parameters and deuterium KIEs for reduction of FMN and haem in wild-type and hinge-modified flavocytochromes $\boldsymbol{b}_{2}$

All experiments were performed at $25^{\circ} \mathrm{C}$ in Tris/HCl buffer, $\mathrm{pH} 7.5(I=0.10 \mathrm{M})$. Stopped-flow data were analysed as described in the Materials and methods section. Values of $k^{\mathrm{F}}$ and $k^{\mathrm{H}}$ are expressed as the number of prosthetic groups reduced per second. Where biphasic or triphasic kinetics were observed, the values reported correspond to the rapid, first phase of reduction, as previously described [12,17]. The upper panel reports the parameters for FMN reduction; typical protein concentrations used were $10 \mu \mathrm{M}$ in the stopped-flow apparatus. The lower panel reports the parameters for haem reduction; typical protein concentrations used were $2.5 \mu \mathrm{M}$ in the stopped-flow apparatus. Abbreviations are as follows: $\left[{ }^{1} \mathrm{H}\right]$ lac, $L-\left[2{ }^{1} \mathrm{H}\right] \mathrm{lactate} ;\left[{ }^{2} \mathrm{H}\right] \mathrm{lac}, \mathrm{L}-\left[2{ }^{2}-{ }^{2} \mathrm{H}\right] \mathrm{lactate}$; $K_{\text {is }}$, substrate inhibition constant. *Rapid phase of triphasic kinetic fit.

\begin{tabular}{|c|c|c|c|c|c|c|c|}
\hline \multirow{2}{*}{$\begin{array}{l}\text { FMN reduction } \\
\text { Enzyme }\end{array}$} & \multicolumn{2}{|l|}{$k^{F}\left(s^{-1}\right)$} & \multicolumn{2}{|l|}{$K_{\mathrm{m}}{ }^{\mathrm{F}}(\mathrm{mM})$} & \multirow[b]{2}{*}{$\mathrm{KIE}$} & \multirow[b]{2}{*}{ Reference } & \\
\hline & {$\left[{ }^{1} \mathrm{H}\right] \mathrm{lac}$} & {$\left[{ }^{2} \mathrm{H}\right] \mathrm{lac}$} & {$\left[{ }^{1} \mathrm{H}\right] \mathrm{lac}$} & {$\left[{ }^{2} \mathrm{H}\right] \mathrm{lac}$} & & & \\
\hline $\begin{array}{l}\text { Wild type } \\
H / 3 \\
H / 6 \\
H \Delta 3 \\
H \Delta 6 \\
H \Delta 9\end{array}$ & $\begin{array}{l}604 \pm 60 \\
541 \pm 25 \\
471 \pm 30 \\
518 \pm 17 \\
514 \pm 21 \\
690 \pm 16\end{array}$ & $\begin{array}{l}75 \pm 5 \\
80 \pm 5 \\
71 \pm 4 \\
71 \pm 30 \\
83 \pm 3 \\
64 \pm 3\end{array}$ & $\begin{array}{l}0.84 \pm 0.20 \\
0.45 \pm 0.10 \\
0.41 \pm 0.08 \\
0.60 \pm 0.08 \\
0.43 \pm 0.08 \\
0.30 \pm 0.03\end{array}$ & $\begin{array}{l}1.33 \pm 0.28 \\
0.18 \pm 0.05 \\
0.13 \pm 0.04 \\
1.46 \pm 0.21 \\
0.30 \pm 0.05 \\
0.44 \pm 0.06\end{array}$ & $\begin{array}{r}8.1 \pm 1.4 \\
6.8 \pm 0.8 \\
6.6 \pm 0.9 \\
7.3 \pm 0.6 \\
6.2 \pm 0.5 \\
10.8 \pm 0.8\end{array}$ & $\begin{array}{l}{[18]} \\
\text { This work } \\
\text { This work } \\
{[11]} \\
{[12]} \\
{[12]}\end{array}$ & \\
\hline Haem reduction & \multicolumn{2}{|l|}{$k^{H}\left(s^{-1}\right)$} & \multicolumn{2}{|l|}{$K_{\mathrm{m}}{ }^{H}(\mathrm{mM})$} & & & \\
\hline Enzyme & {$\left[{ }^{1} \mathrm{H}\right] \mathrm{lac}$} & $\left.{ }^{2} \mathrm{H}\right] \mathrm{lac}$ & {$\left[{ }^{1} \mathrm{H}\right] \mathrm{lac}$} & $\left.{ }^{2} \mathrm{H}\right] \mathrm{lac}$ & $K_{\text {is }}(\mathrm{mM})$ & KIE & Reference \\
\hline $\begin{array}{l}\text { Wild type } \\
H / 3^{*} \\
H / 6^{*} \\
H \Delta 3 \\
H \Delta 6^{*} \\
H \Delta 9^{*}\end{array}$ & $\begin{array}{c}445 \pm 50 \\
29 \pm 3 \\
14 \pm 2 \\
91 \pm 3 \\
29 \pm 1 \\
9 \pm 1\end{array}$ & $\begin{array}{r}71 \pm 5 \\
14 \pm 2 \\
8 \pm 1 \\
32 \pm 1 \\
15 \pm 1 \\
8 \pm 1\end{array}$ & $\begin{array}{l}0.53 \pm 0.05 \\
0.07 \pm 0.03 \\
0.04 \pm 0.02 \\
0.38 \pm 0.07 \\
0.04 \pm 0.01 \\
0.07 \pm 0.02\end{array}$ & $\begin{array}{l}0.68 \pm 0.05 \\
0.07 \pm 0.02 \\
0.02 \pm 0.01 \\
0.80 \pm 0.13 \\
0.10 \pm 0.02 \\
0.21 \pm 0.06\end{array}$ & $\begin{array}{l}174 \pm 8 \\
114 \pm 26 \\
178 \pm 121 \\
132 \pm 8 \\
- \\
-\end{array}$ & $\begin{array}{l}6.3 \pm 1.2 \\
2.1 \pm 0.6 \\
1.8 \pm 0.5 \\
2.8 \pm 0.2 \\
1.9 \pm 0.2 \\
1.1 \pm 0.2\end{array}$ & $\begin{array}{l}{[18]} \\
\text { This work } \\
\text { This work } \\
{[11]} \\
{[12]} \\
{[12]}\end{array}$ \\
\hline
\end{tabular}


Table 2 Steady-state turnover number for reduction of ferricyanide by wild-type and hinge-modified flavocytochromes $\boldsymbol{b}_{2}$

All experiments were performed at $25^{\circ} \mathrm{C}$ in $10 \mathrm{mM}$ Tris/HCl buffer, pH $7.5(/=0.10 \mathrm{M})$. Saturating concentrations of ferricyanide were used: for wild-type enzyme, [ferricyanide] = $1 \mathrm{mM}$; for $\mathrm{H} \Delta 3$ enzyme, $2 \mathrm{mM}$; for $\mathrm{H} \Delta 6, \mathrm{H} \Delta 9, \mathrm{H} / 3$ and $\mathrm{H} / 6$ enzymes, $8 \mathrm{mM}$. The $k_{\text {cat }}$ values are expressed as moles of electrons transferred per second per mole of enzyme (as L-lactate is a two-electron donor, these values can be halved to express them in terms of moles of substrate reduced per second). The standard error was $10 \mathrm{~s}^{-1}$ in all cases.

\begin{tabular}{lll}
\hline Enzyme & $k_{\text {cat }}\left(s^{-1}\right)$ & Reference \\
\hline$W T$ & 400 & {$[17]$} \\
$H / 3$ & 400 & This work \\
$H / 6$ & 400 & This work \\
$H \Delta 3$ & 257 & {$[11]$} \\
$H \Delta 6$ & 276 & {$[12]$} \\
$H \Delta 9$ & 400 & {$[12]$} \\
\hline
\end{tabular}

experimental error, as the value for wild-type flavocytochrome $b_{2}$ (Table 1, top panel). This is also true of the hinge-deletion enzymes [11,12]. Indeed, for all the hinge-modified enzymes, the rate constants for FMN reduction are within $20 \%$ of the value for wild-type flavocytochrome $b_{2}$. The apparent $K_{\mathrm{m}}$ for presteady-state flavin reduction $\left(K_{\mathrm{m}}{ }^{\mathrm{F}}\right)$ values for the hinge-modified enzymes are lower than that for the wild-type enzyme (except for $\mathrm{H} \Delta 3$, which is the same as wild-type, within experimental error). However, the largest fall in $K_{\mathrm{m}}{ }^{\mathrm{F}}$ is only about 2-fold. All of the deuterium KIE values for the hinge-modified enzymes are the same within experimental error as that reported for wild-type flavocytochrome $b_{2}$, implying that the rate-limiting step for FMN reduction in all the hinge-modified enzymes is hydrogen abstraction from C-2 of L-lactate, as it is in wild-type flavocytochrome $b_{2}$ [20]. This remarkable consistency in data for FMN reduction between the five hinge-modified enzymes and wild-type flavocytochrome $b_{2}$ implies that changing the length of the interdomain hinge region, either by truncation or elongation, has no significant effect upon the lactate dehydrogenase function of the enzyme.

It is worth noting that for the hinge-insertion enzymes, the FMN reduction traces fitted very well to either monophasic or biphasic kinetics, with the rate constant for the first phase of the biphasic fit being similar to that of the monophasic fit. The data reported in Table 1 (top panel) for these enzymes are from fits to a monophasic exponential equation. Similar behaviour has been reported for another hinge mutant of flavocytochrome $b_{2}$, termed hinge-swap [9]. This situation is in contrast to that observed for the hinge-deletion and wild-type enzymes, where clearly better fits were obtained with biphasic kinetics, at least at higher lactate concentrations [9]. For the wild-type enzyme, it is known that the rapid first phase corresponds to the initial reduction of FMN (and haem) and the slow phase is due to disproportionation between subunits within the flavocytochrome $b_{2}$ tetramer and subsequent re-reduction of the flavin (see below). This reaction is catalytically irrelevant during steady-state turnover [20].

The apparent lack of effect of hinge-modification on the dehydrogenase function of flavocytochrome $b_{2}$ was also observed in steady-state experiments when ferricyanide was used as electron acceptor. Table 2 reports the steady-state turnover numbers $\left(k_{\text {cat }}\right)$ for the hinge-insertion flavocytochromes $b_{2}$ when $\mathrm{L}-\left[2-{ }^{1} \mathrm{H}\right]$ lactate was used as a substrate and ferricyanide was used as the electron acceptor. These results are compared with previously reported values for the wild-type and hinge-deletion enzymes. The other kinetic parameters $\left(K_{\mathrm{m}}\right.$ and KIE) are not

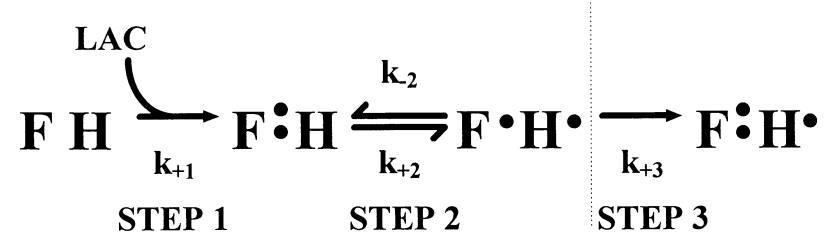

Scheme 2 The electron-transfer processes which occur in a single flavocytochrome $b_{2}$ subunit upon full reduction of the enzyme by L-lactate

Step 1, reduction of FMN; step 2, FMN $\rightarrow$ haem electron transfer; step 3, entry of the third electron per subunit to generate fully reduced enzyme. The origin of step 3 is described in detail in the text. F, FMN; H, haem; black dots denote electrons.

described, as they are very similar to wild-type flavocytochrome $b_{2}$ for all the hinge-modified enzymes $[11,12,17]$. Ferricyanide can accept electrons from both the haem and FMN prosthetic groups and the ability of flavocytochrome $b_{2}$ to donate electrons to ferricyanide is a measure of its L-lactate dehydrogenase capability. From Table 2, it is evident that all the hinge-modified enzymes are good L-lactate dehydrogenases, comparable with the wild-type enzyme. The largest decrease in $k_{\text {cat }}$ is observed for the $\mathrm{H} \Delta 3$ and $\mathrm{H} \Delta 6$ enzymes ( $60 \%$ of the wild-type value), and in $\mathrm{H} \Delta 9$ and both hinge-insertion enzymes, $k_{\text {cat }}$ is the same within experimental error as for wild-type flavocytochrome $b_{2}$ (Table 2).

\section{Effect of the hinge-modifications on intraprotein electron transfer}

Investigation by pre-steady-state kinetics of haem reduction

Kinetic parameters for pre-steady-state haem reduction by L-[2$\left.{ }^{1} \mathrm{H}\right]$ lactate and $\mathrm{L}-\left[2-{ }^{2} \mathrm{H}\right]$ lactate for wild-type and hinge-modified flavocytochromes $b_{2}$ are reported in Table 1 (lower panel). The hinge-insertion enzymes $\mathrm{H} I 3$ and $\mathrm{H} I 6$ have rate constants for haem reduction by L-lactate that are 15 - and 30 -fold lower respectively than the corresponding value for the wild-type enzyme. Thus, as the hinge region is progressively lengthened, the rate constant for haem reduction decreases in value. This is analogous to the situation with the hinge-deletion enzymes, where progressive truncation of the hinge region causes a concomitant decrease in the rate constant for haem reduction $([11,12]$ and Table 1 , lower panel). It can therefore be concluded that these changes in the length of the interdomain hinge region result in severe disruption of haem reduction.

Scheme 2 illustrates the kinetic processes that occur upon haem reduction by L-lactate under pre-steady-state conditions. The rapid phase of haem reduction by L-lactate arises from a two-step process in which two electrons from L-lactate enter a flavocytochrome $b_{2}$ subunit at the level of the FMN and are then redistributed between the FMN and the haem in each subunit $[21,22]$. The slower, second phase is mainly due to the entry of a third electron per subunit. Flavocytochrome $b_{2}$ is tetrameric and when fully reduced under pre-steady-state conditions in the absence of an external electron acceptor, it can 'hold' twelve electrons: three per subunit, two on the FMN and one on the haem. Upon reduction of the fully oxidized enzyme by excess lactate ( $>$ six molecules per tetramer), the enzyme will eventually become fully reduced. Initially, four lactate molecules will bind, one to each subunit, reducing the enzyme to the eight electron level. Electron redistribution by intersubunit electron transfer allows further reduction by an additional two lactate molecules, 
resulting in full reduction of the tetramer [8]. At the single subunit level, this is manifested as entry of a third electron $[21,22]$. This slower phase is catalytically irrelevant during flavocytochrome $b_{2}$ turnover, when the enzyme behaves as a twoelectron transferase.

The reported rate constant for haem reduction of the wild-type enzyme by L-lactate is $445 \pm 50 \mathrm{~s}^{-1}$ (Table 1, lower panel), measured from the rapid phase of a biphasic fit to the haem reduction trace [17]. However, fitting these data to biphasic kinetics is an approximation. Step 2 in Schemes 1 and 2 is the first $\mathrm{FMN} \rightarrow$ haem electron transfer, generating flavin semiquinone and reduced haem. This step is actually faster than the preceding step 1 (FMN reduction), so that the apparent rate constant for haem reduction by L-lactate will be limited by the rate of formation of reduced FMN. If these factors are taken into account and the data fitted as described in [22], a rate constant of $1500 \pm 500 \mathrm{~s}^{-1}$ is obtained. This describes the rate of electron transfer from fully reduced FMN $\rightarrow$ haem. Reassuringly, the same rate constant, within experimental error, for this step has been directly measured by laser flash photolysis techniques, where a value of $1900 \pm 100 \mathrm{~s}^{-1}$ was obtained [23]. In the case of hinge-modified flavocytochromes $b_{2}, \mathrm{FMN}$ reduction is much faster than the subsequent haem reduction rate and no appreciable time lag occurs before sufficient reduced FMN is formed prior to haem reduction. So, from fitting the haem reduction traces to biphasic or triphasic kinetics at saturating Llactate concentrations, one can obtain the rate constant for the rapid, first phase of the trace. This represents the rate constant $\left(k_{\text {obs }}\right)$ for the attainment of equilibrium between the FMN and haem and is thus the sum of the two microscopic steps $k_{+2}$ and $k_{-2}$ in step 2 of Scheme $2\left(\mathrm{FMNH}_{2} \rightarrow\right.$ haem electron transfer). However, because the equilibrium lies $85 \%$ in favour of reduced haem in the wild-type enzyme [24], and also, we believe, in the hinge-modified enzymes (see later), and because the rate constants for the hinge-modulated enzymes are much lower than that for the wild-type enzyme, $k_{\text {obs }}$ is effectively equivalent to $k_{+2}$. Thus, in the hinge-modified flavocytochromes $b_{2}$, the actual rate constants for fully reduced FMN $\rightarrow$ haem electron transfer are 50- and 100-fold lower for the hinge-insertion enzymes and 16-, 50- and 170-fold lower for the hinge-deletion enzymes [11,12] respectively compared with the value for wild-type flavocytochrome $b_{2}$. These data clearly show that maintaining the structural integrity of the interdomain hinge region is crucial for efficient intraprotein electron transfer. The larger the truncation or elongation of the hinge region, the greater the decrease in magnitude of the rate constant for fully reduced FMN $\rightarrow$ haem electron transfer. This dramatic effect is illustrated graphically in Figure 3.

The apparent $K_{\mathrm{m}}$ for pre-steady-state haem reduction $\left(K_{\mathrm{m}}{ }^{\mathrm{H}}\right)$ values of the hinge-modified enzymes are all lower than the corresponding value for the wild-type enzyme (Table 1, lower panel). These decreases are due to the fact that pre-steady-state haem reduction involves several kinetic steps, as described above. The fall in the rate constants for haem reduction in the hingemodified enzymes are also manifested as a decrease in the $K_{\mathrm{m}}$. These $K_{\mathrm{m}}$ values are not a reflection of the $K_{\mathrm{d}}$ for L-lactate binding. The lactate dehydrogenation step is unaffected by the hinge modifications and $K_{\mathrm{d}}$ will not be affected by events following FMN reduction.

The deuterium KIE values for haem reduction by L-lactate for all the hinge-modified enzymes are significantly lower than that for wild-type flavocytochrome $b_{2}$ (Table 1, lower panel). This implies that for these mutants, hydrogen abstraction from C-2 of L-lactate contributes much less to rate limitation of haem reduction than it does in the wild-type enzyme. Essentially, the

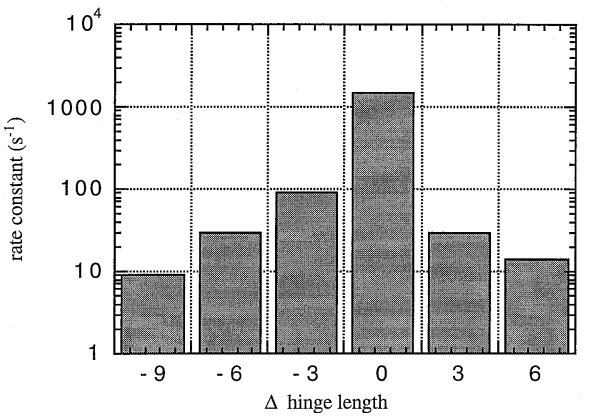

Figure 3 Graphical representation of the effect of hinge-length modulation upon intraprotein electron transfer

The rate constant for fully reduced FMN $\rightarrow$ haem electron transfer is plotted on a logarithmic scale against the degree of hinge truncation or elongation.

KIE data are consistent with the view that intraprotein electron transfer has been severely disrupted in the hinge-modified enzymes.

As discussed previously [12], the hinge-deletion enzymes ( $\mathrm{H} \Delta 6$ and $\mathrm{H} \Delta 9$ ) and both the hinge-insertion enzymes (as described here) have haem reduction traces that fit better to triphasic kinetics, as opposed to biphasic kinetics for the $\mathrm{H} \Delta 3$ enzyme $[11,12]$ and the fitting procedure described by Chapman et al. for wild-type flavocytochrome $b_{2}$ [22]. For the hinge-insertion enzymes, the first phase typically comprised about $30-40 \%$ of the total amplitude of the trace over most of the L-lactate concentrations investigated. The other two phases that were fitted are too slow to be kinetically relevant in the catalytic cycle and as such will not be discussed here. For a full explanation of these phases see Sharp et al. [12].

\section{Investigation by steady-state cytochrome $c$ reduction}

Table 3 presents the data for steady-state cytochrome $c$ reduction by the hinge-insertion flavocytochromes $b_{2}$, using L- $\left[2-{ }^{1} \mathrm{H}\right]$ lactate and $\mathrm{L}-\left[2-{ }^{2} \mathrm{H}\right]$ lactate as substrates. These results are compared with previously reported values for the wild-type and hingedeletion enzymes. In contrast to the kinetic data with ferricyanide, there are significant differences between the wild-type and hingemodified enzymes when cytochrome $c$ is used as the electron acceptor. The $k_{\text {cat }}$ values are decreased by factors of 7 and 16 in the hinge-insertion enzymes $\mathrm{H} I 3$ and $\mathrm{H} I 6$ respectively compared with the value for the wild-type enzyme (Table 3 ). This situation is similar to that observed with the hinge-deletion enzymes, where progressive truncation of the hinge region causes the $k_{\text {eat }}$ for cytochrome $c$ reduction to fall in value. Clearly, modulation of the length of the flavocytochrome $b_{2}$ interdomain hinge region results in severely impaired cytochrome $c$ reductase activity. The deuterium KIEs for all the hinge-modified enzymes are lower than that for wild-type flavocytochrome $b_{2}$ when cytochrome $c$ is used as the electron acceptor (Table 3 ). This implies that electrontransfer reactions subsequent to hydrogen abstraction from C-2 of L-lactate contribute to overall rate limitation to a greater extent in these enzymes than in wild-type flavocytochrome $b_{2}$.

Interestingly, the hinge-insertion enzymes exhibit severe substrate inhibition at relatively low L-lactate concentrations, with inhibition constants that are the same within the experimental error (about $8 \mathrm{mM}$; Table 3), despite the different degree of hinge-lengthening. This inhibition is the strongest observed for all the hinge-modified enzymes and may reflect 


\section{Table 3 Steady-state kinetic parameters and deuterium KIEs for reduction of wild-type and hinge-modified flavocytochromes $\boldsymbol{b}_{2}$ by cytochrome $\boldsymbol{c}$}

All experiments were performed at $25^{\circ} \mathrm{C}$ in $10 \mathrm{mM}$ Tris/ $\mathrm{HCl}$ buffer, $\mathrm{pH} 7.5(/=0.10 \mathrm{M})$. Concentrations of cytochrome $c$ electron acceptor were: wild-type enzyme, $30 \mu \mathrm{M}$ (> $75 \%$ saturating): $\mathrm{H} \Delta 3, \mathrm{H} \Delta 6$ and $\mathrm{H} \Delta$ 9 enzymes, $35 \mu \mathrm{M}$ ( $>80 \%$ and $>90 \%$ saturating for $\mathrm{H} \Delta 3$ and $\mathrm{H} \Delta 6 / \mathrm{H} \Delta 9$ respectively); $\mathrm{H} / 3$ and $\mathrm{H} / 6$ enzymes, $50 \mu \mathrm{M}$ (> $90 \%$ saturating in both cases). The $k_{\text {cat }}$ values are expressed as moles of electrons transferred per second per mole of enzyme (as L-lactate is a two-electron donor, these values can be halved to express them in terms of moles of substrate reduced per second). Errors quoted represent standard deviations from a non-linear least-squares fit. Abbreviations are as follows: $\left[{ }^{1} \mathrm{H}\right] \mathrm{lac}, \mathrm{L}-\left[2{ }^{-1} \mathrm{H}\right] \mathrm{lactate} ;\left[{ }^{2} \mathrm{H}\right] \mathrm{lac}, \mathrm{L}-\left[2{ }^{-2} \mathrm{H}\right] \mathrm{lactate} ; K_{\mathrm{is}}$, substrate inhibition constant.

\begin{tabular}{|c|c|c|c|c|c|c|c|}
\hline \multirow[b]{2}{*}{ Enzyme } & \multicolumn{2}{|l|}{$k_{\text {cat }}\left(\mathrm{s}^{\star-1}\right)$} & \multicolumn{2}{|l|}{$K_{\mathrm{m}}(\mathrm{mM})$} & \multirow[b]{2}{*}{$K_{\text {is }}(\mathrm{mM})$} & \multirow[b]{2}{*}{$\mathrm{KIE}$} & \multirow[b]{2}{*}{ Reference } \\
\hline & {$\left[{ }^{1} \mathrm{H}\right] \mathrm{lac}$} & {$\left[{ }^{2} \mathrm{H}\right] \mathrm{lac}$} & {$\left[{ }^{1} \mathrm{H}\right] \mathrm{lac}$} & {$\left[{ }^{2} \mathrm{H}\right] \mathrm{lac}$} & & & \\
\hline Wild type & $207 \pm 10$ & $70 \pm 10$ & $0.24 \pm 0.04$ & $0.48 \pm 0.10$ & $173 \pm 13$ & $3.0 \pm 0.6$ & [18] \\
\hline & $28.3 \pm 0.7$ & $15.9 \pm 0.7$ & $>0 . \overline{10}$ & $0.10 \pm 0.03$ & $7.4 \pm 0.6$ & $1.8 \pm 0.1$ & This work \\
\hline $\mathrm{H} / 6$ & $12.7 \pm 0.3$ & $7.1 \pm 0.4$ & $>0.10$ & $>0.10$ & $8.9 \pm 0.8$ & $1.8 \pm 0.1$ & This work \\
\hline $\mathrm{H} \Delta 3$ & $39 \pm 1$ & $20 \pm 1$ & $0.25 \pm 0.03$ & $0.61 \pm 0.07$ & $92 \pm 5$ & $2.0 \pm 0.1$ & [11] \\
\hline $\mathrm{H} \Delta 6$ & $33.5 \pm 1.4$ & $14 \pm 0.4$ & $0.09 \pm 0.01$ & $0.13 \pm 0.02$ & $21.1 \pm 2.2$ & $2.4 \pm 0.2$ & [12] \\
\hline $\mathrm{H} \Delta 9$ & $7.9 \pm 0.4$ & $4.6 \pm 0.3$ & $0.02 \pm 0.01$ & N.D. & $14.0 \pm 0.2$ & $1.7 \pm 0.2$ & [12] \\
\hline
\end{tabular}

greater accessibility of the substrate to the active site for the hinge insertion, in contrast to the $\mathrm{H} \Delta 3$ and wild-type enzymes and to a lesser extent the $\mathrm{H} \Delta 6$ and $\mathrm{H} \Delta 9$ enzymes.

The region of Scheme 1 labelled 'cyt $c$ ' represents steady-state cytochrome $c$ turnover by flavocytochrome $b_{2}$ in the presence of saturating or optimal concentrations of L-lactate. The rate constants for cytochrome $c$ reduction by wild-type and hingemodified flavocytochromes $b_{2}$ are also reported in the Scheme. A striking observation that can be made for all the enzymes, except $\mathrm{H} \Delta 3$ and wild-type flavocytochrome $b_{2}$, is that the rate constants for step 2, fully reduced FMN $\rightarrow$ haem electron transfer, are the same within experimental error as that for steady-state cytochrome $c$ reduction. This implies that, in the case of the former group of enzymes, intraprotein electron transfer from fully reduced FMN $\rightarrow$ haem is totally rate limiting for enzyme turnover. For the wild-type enzyme, it is known that the major rate-limiting step in the catalytic cycle is at step 4, which is the second intraprotein electron transfer step from flavin semiquinone $\rightarrow$ haem [25]. This is also likely to contribute substantially to rate limitation in the $\mathrm{H} \Delta 3$ enzyme.

These conclusions are strongly supported by the deuterium KIE values for haem and cytochrome $c$ reduction by L-lactate. All the hinge-modified enzymes where fully reduced FMN $\rightarrow$ haem electron transfer is totally rate limiting have KIE values which are the same within experimental error for both presteady-state haem reduction and steady-state cytochrome $c$ reduction.

\section{Haem redox mid-point potential of hinge-modified enzymes}

We considered the possibility that mutation, in this case by insertions, of the hinge region of flavocytochrome $b_{2}$ might affect the redox potentials of the prosthetic groups. An increase and/or a decrease of the mid-point potentials of the FMN and haem prosthetic groups respectively would lower the thermodynamic driving force for electron transfer, which could cause the rate of $\mathrm{FMN} \rightarrow$ haem electron transfer to decrease in value. There is clearly no, or very little, effect upon the mid-point potentials of the FMN oxidized/reduced couple for the two hinge-insertion enzymes, as the microscopic rate constants for FMN reduction are not significantly different from the wild-type enzyme (Table 1 , lower panel). However, there is a possibility that a large change in the haem mid-point potential could contribute to the lowering of the rate constants for haem reduction in the hingeinsertion enzymes. To discount this proposal, we measured the haem mid-point potentials for the HI3 and HI6 enzymes. Both had the same potential within experimental error as wild-type flavocytochrome $b_{2} ; E_{\mathrm{m}}(\mathrm{H} / 3)=-22 \pm 5 \mathrm{mV}, E_{\mathrm{m}} \quad(\mathrm{H} I 6)=$ $-26 \pm 5 \mathrm{mV}$ and $E_{\mathrm{m}}$ (wild type) $=-17 \pm 3 \mathrm{mV}$ [26]. This implies that the decreased rate of intraprotein electron transfer is due to modulation of the hinge region alone and not to any change in the redox chemistry of the prosthetic groups.

\section{Conclusion}

From our studies on the hinge-modified flavocytochromes $b_{2}$, we draw the following conclusions. (1) Modification of the length of the interdomain hinge region has little influence on the lactate dehydrogenase activity of the enzyme. (2) Structural integrity of the hinge region is crucial in mediating efficient intraprotein electron transfer from FMN $\rightarrow$ haem. It is apparent that the magnitude of the rate constant for haem reduction is less affected by an equivalent size of deletion in the hinge region, compared with an insertion. For example, the rate constant for haem reduction by the $\mathrm{H} \Delta 3$ enzyme is 3 -fold higher than that for $\mathrm{H} I 3$ flavocytochrome $b_{2}$ (Table 1, lower panel). This is an empirical observation and any quantitative information derived from such data requires that the structural changes imposed by such mutations be known. (3) In the enzymes $\mathrm{H} I 3, \mathrm{H} I 6, \mathrm{H} \Delta 6$ and $\mathrm{H} \Delta 9$, the major rate-limiting step is flavin hydroquinone $\rightarrow$ haem electron transfer, this is the first intraprotein electron transfer event. However, in the wild-type and $\mathrm{H} \Delta 3$ enzymes, the major rate-limiting step is flavin semiquinone $\rightarrow$ haem electron transfer, the second intraprotein electron transfer event. (4) The hinge modifications have very little effect on the redox potentials of the prosthetic groups.

We would like to thank Professor F. Scott Mathews and Drs. Florence Lederer, Mariella Tegoni and Christian Cambillau for helpful discussions. This work was funded by the Biotechnology and Biological Sciences Research Council and the Engineering and Physical Sciences Research Council. We thank the Commission of the European Communities for support (FLAPS Network; Contract no. ERBCHRXCT930166).

\section{REFERENCES}

1 Daum, G., Böhni, P. C. and Schatz, G. (1982) J. Biol. Chem. 257, 13028-13033

2 Appleby, C. A. and Morton, R. K. (1954) Nature (London) 173, 749-752

3 Jacq, C. and Lederer, F. (1974) Eur. J. Biochem. 41, 311-320 
4 Xia, Z.-X. and Mathews, F. S. (1990) J. Mol. Biol. 212, 837-863

5 Kraulis, P. J. (1991) J. Appl. Crystallogr. 24, 946-950

6 Ogura, Y. and Nakamura, T. (1966) J. Biochem. (Tokyo) 60, 77-86

7 Capeillère-Blandin, C., Iwatsubo, M., Testylier, G. and Labeyrie, F. (1980) in Flavins and Flavoproteins (Yagi, K. and Yamamoto, T., eds.), pp. 617-630, Japan Scientific Societies Press, Tokyo

8 Balme, A., Brunt, C. E., Pallister, R. L., Chapman, S. K. and Reid, G. A. (1995) Biochem. J. 309, 601-605

9 White, P., Manson, F. D. C., Brunt, C. E., Reid, G. A. and Chapman, S. K. (1993) Biochem. J. 291, 89-94

10 Labeyrie, F., Beloeil, J. C. and Thomas, M. A. (1988) Biochim. Biophys. Acta 953 $134-141$

11 Sharp, R. E., White, P., Chapman, S. K. and Reid, G. A. (1994) Biochemistry 33, $5115-5120$

12 Sharp, R. E., Chapman, S. K. and Reid, G. A. (1996) Biochemistry 35, 891-899

13 Kunkel, T. A. (1985) Proc. Natl. Acad. Sci. U.S.A. 82, 488-492

14 Sambrook, J., Fritsch, E. F. and Maniatis, T. (1989) Molecular Cloning: A Laboratory Manual, 2nd edn., Cold Spring Harbor Laboratory Press, Cold Spring Harbor, New York

Received 1 December 1995/22 January 1996; accepted 26 January 1996
15 Black, M. T., White, S. A., Reid, G. A. and Chapman, S. K. (1989) Biochem. J. 258 255-259

16 Pajot, P. and Groudinsky, O. (1970) Eur. J. Biochem. 12, 158-164

17 Miles, C. S., Rouvière-Fourmy, N., Lederer, F., Mathews, F. S., Reid, G. A., Black, M. T. and Chapman, S. K. (1992) Biochem. J. 285, 187-192

18 Pompon, D., Iwatsubo, M. and Lederer, F. (1980) Eur. J. Biochem. 104, 479-488

19 Dutton, P. L. (1978) Methods Enzymol. 54, 422-435

20 Pompon, D. (1980) Eur. J. Biochem. 106, 151-159

21 Capeillère-Blandin, C., Bray, R. C., Iwatsubo, M. and Labeyrie, F. (1975) Eur. J. Biochem. 54, 549-566

22 Chapman, S. K., Reid, G. A., Daff, S., Sharp, R. E., White, P., Manson, F. D. C. and Lederer, F. (1994) Biochem. Soc. Trans. 22, 713-718

23 Hazzard, J. T., McDonough, C. A. and Tollin, G. (1994) Biochemistry 33 13445-13454

24 Walker, M. C. and Tollin, G. (1991) Biochemistry 30, 5546-5555

25 Daff, S., Ingledew, W. J., Reid, G. A. and Chapman, S. K. (1996) Biochemistry, in the press

26 Miles, C. S. (1992) Ph.D. Thesis, University of Edinburgh 